\title{
Pattern of Indirect Immunofluorescence Assay Antinuclear Antibody in Pediatric Lupus Nephritis
}

\author{
Fadhila Novianti, ${ }^{1}$ Reni Ghrahani, ${ }^{2}$ Noormarina Indraswari ${ }^{3}$ \\ ${ }^{1}$ Faculty of Medicine Universitas Padjadjaran, Bandung, Indonesia \\ ${ }^{2}$ Departement of Child Health, Faculty of Medicine Universitas Padjadjaran-Dr. Hasan Sadikin Hospital, \\ Bandung, Indonesia \\ ${ }^{3}$ Department of Public Health, Faculty of Medicine Universitas Padjadjaran, Bandung, Indonesia
}

\begin{abstract}
Objective: To determine the association between Anti-Nuclear Antibody (ANA) pattern in pediatric Systemic Lupus Erythematosus (SLE) patients and proteinuria as a sign of renal involvement in SLE.
\end{abstract}

\begin{abstract}
Methods: This was a cross-sectional study, using data from medical records involving 89 newly diagnosed with SLE (aged $\leq 18$ years) in Department of Child Health Dr. Hasan Sadikin General Hospital, Bandung, from January 1, 2018 to June 30, 2019. Data of ANA pattern and proteinuria were collected from medical record. ANA pattern was examined by Immunofluorescence Assay (IFA) method. Chi-square was used to analyze the association between ANA pattern and proteinuria as a sign of renal involvement in pediatric SLE patients.
\end{abstract}

pISSN: 2302-1381;

eISSN: 2338-4506;

http://doi.org/10.15850/

ijihs.v8n1.1911

IJIHS. 2020;8(1):38-42

Received:

January 3, 2020

Accepted:

May 21, 2020
Results: There were 89 patients, consisting of 7 male (7.9\%) and 82 female $(92 . \%)$ with median age of $14(\mathrm{IQR}=12-16)$. There were only 44 pattern of ANA as follow: homogenous $56.8 \%$, speckled $36.4 \%$, and nucleolar $6.8 \%$. However, there is no significant association between homogenous pattern with proteinuria events $(\mathrm{p}=0.831)$.

Conclusions: Homogenous pattern was the most frequent ANA pattern in children with SLE, and the pattern has no association with proteinuria events.

Keywords: Antinuclear antibody pattern, indirect immunofluorescence assay; proteinuria, systemic lupus erythematosus

\section{Introduction}

Systemic Lupus Erythematosus (SLE) is a chronic autoimmune disease characterized by a loss of tolerance from the immune system and formed autoantibodies. The autoantibody also known as Anti-Nuclear Antibody (ANA) which have many subtypes that can attack self-antigens and cause inflammation in several organs, such as skin, joints, kidneys, blood-forming cells, blood vessels, and central nervous system. ${ }^{1,2,3,4}$ ANA test is one of tests that used for helping diagnose autoimmune

Correspondence:

Fadhila Novianti,

Faculty of Medicine Universitas Padjadjaran

e-mail: fadhilanovianti@gmail.com disorder. Patients with SLE are almost always positive for ANA, meanwhile patients with other autoimmune disoreders who have positive ANA result are varies, even a significant number patients with other types of disorders and some healthy people might have positive ANA, especially at low levels. Moreover, American College of Rheumatology (ACR) 1997 recommends Immunoflourecence Assay (IFA) method as a gold standard of ANA test which can help clinician to diagnose the autoimmune disorder more specifically besides another manifestations showed by the patient. It can also help to reduce risk of false positive or errorneous. ${ }^{1}$ By the result of the test, the clinician might see the titers and different types of ANA through the staining pattern. Based on International Consensus of ANA Pattern (ICAP), ANA is divided into 
three major categories, namely nuclear (such as homogeneous, speckled, centromere, nucleardots and nucleolar), cytoplasmic and mitotic patterns. The results of the titer and ANA pattern will be varied in each patients. ${ }^{7,15,16}$

Systemic Lupus Erythematosus can occur in both adults and children. However, when compared with adults, children and adolescents have a higher risk of severity and involvement of other organs as a manifestation in SLE. ${ }^{5,6}$ The most common and difficult manifestation of SLE is renal involvement. The involvement of the renal as a clinical manifestation will cause inflammation in the renal called nephritis. ${ }^{5,6,8}$ One of the result of the inflammation is the presence of protein in the urine, which called proteinuria. In the case of pediatric SLE, lupus nephritis is more common in females than males with a ratio of 8-13: 1 and in the age range of 14-16 years. ${ }^{5}$ Also, pediatric SLE with nephritis having poorer prognosis than others without nephritis. ${ }^{8,9}$

The study about relevance of ANA in SLE has been adressed repeteadly. Through the studies, ANA is known to have a correlation with the clinical manifestations of SLE, especially in patient with positive anti-

\section{Table 1 Characteristics of Systemic Lupus} Erythematosus Patients

\begin{tabular}{lc}
\hline \multicolumn{1}{c}{ Characteristics } & Total (n=89) \\
\hline $\begin{array}{l}\text { Age (years) } \\
\text { Median [IQR] }\end{array}$ & $14(12-16)$ \\
Gender (n, \%) & $7(7.9 \%)$ \\
$\quad$ Boys & $82(92.1 \%)$ \\
$\quad$ Girls & \\
Skin manifestation (n, \%) & $32(44.4 \%)$ \\
$\quad$ Malar Rash & $14(22.6 \%)$ \\
$\quad$ Discoid Rash & $29(36.7 \%)$ \\
$\quad$ Photosensitivity & $12(19.7 \%)$ \\
Mouth Ulcers & \\
Renal manifestation (n, \%) & $46(51.7 \%)$ \\
$\quad$ Proteinuria & $42(47.2 \%)$ \\
$\quad$ Hematuria & \\
Hematological manifestation & \\
(n, \%) & \\
$\quad$ Anemia & $61(77.2 \%)$ \\
Leukopenia & $19(24.1 \%)$ \\
Thrombocytopenia & \\
Neuropsychiatric & \\
manifestation (n, \%) & \\
\hline
\end{tabular}

dsDNA and/or anti-Sm. The presence of anti-dsDNA was found to be associated with renal disorder, haematological involvemnet and serositis, meanwhile patient with antiSm had the highest frequency of malar rash, oral ulcers, arthritis, and serositis. ${ }^{18}$ Whereas, studies about ANA pattern and its relation with the clinical manifestation of SLE are very scarce especially proteinuria. A few studies stated, there were no association between ANA Pattern and clinical manifestation of SLE, Frodlund $M$ et al. observed that some pattern may have an association with the clinical manifestation of SLE. ${ }^{10,12,13,14}$ However, this finding needs more confirmation by others. ${ }^{10,14,17,18}$

This study is aimed to determine the association between ANA pattern in pediatric SLE patients and proteinuria as a sign of renal involvement in Department of Child Health Hasan Sadikin Bandung General Hospital.

\section{Methods}

This was a cross-sectional study conducted of 89 subejct newly diagnosed patients with Systemic Lupus Erythematosus (aged $\leq 18$ years), in Department of Child Health Dr. Hasan Sadikin General Hospital, Bandung, from January $1^{\text {st }} 2018$ until June $30^{\text {th }} 2019$. Secondary data from medical records of newly diagnosed with SLE were used in this study. Data of age, gender, ANA pattern, and proteinuria were collected from the medical records. ANA pattern was examined by Immunoflourecence Assay Method. Data was taken after obtaining Ethical Clearance issued by Health Research Ethics Committee of Universitas Padjadjaran Bandung No. 719/ UN6.KEP/EC/2019 and Research Licensing Letter issued by Research Ethics Committee of Dr. Hasan Sadikin General Hospital Bandung No. LB.02.01/X.2.2.1/10047/2019. Microsoft $\AA$ Excel 2013 and IBM $\AA$ SPSS $\AA$ Version 20 were used to process the data. Chisquare was used to analyze the association between ANA pattern and proteinuria as a sign of renal involvement in SLE and $p<0.05$ was considered statistically significant.

\section{Results}

There were 89 subjects, consisting of 7 male and 82 female with median age of 14 (IQR $=12-$ 16) (see Table 1). Besides hematological manifestation, renal was the most frequent manifestation in pediatric SLE patients (see Table 1). Pattern of ANA was analyzed and 
Table 2 ANA Pattern of Systemic Lupus Erythematosus Patients

\begin{tabular}{lc}
\hline \multicolumn{1}{c}{ Category } & Total (n=44) \\
\hline Homogenous (n, \%) & $25(56.8 \%)$ \\
Speckled (n, \%) & $16(36.4 \%)$ \\
Nucleolar (n, \%) & $3(6.8 \%)$ \\
\hline
\end{tabular}

homogenous was the most common type followed by speckled, and nucleolar (Table 2 ). Further data processing was performed with chi-square to assess the association between ANA pattern and proteinuria events as a sign of renal involvement in pediatric SLE patients (Table 3). There is no significant association between homogenous pattern with proteinuria events.

\section{Discussion}

Based on the study, it is known that the subjects are mostly girls. Similar with the result of other studies that have been done, one of them is study from Almaani S, et al. which stated that the most of children with SLE were girls than boys, with female-to-male ratio range between 8-13:1 and in the age range of 14-16 years. ${ }^{5}$ This finding was because of hormonal factors such as sex steroids which are owned by girls, thus will increasing the risk of SLE. ${ }^{1,6}$

In patients with SLE, we can find the presence of ANA. ANA is autoantibodies which formed by the immune system that can attack self-antigen and cause inflammation in many tissues and organs. . $^{1,2,3,4,5}$ ANA test is a primary test to determines the presence of ANA. Despite this, ACR 1997 recommends Immunoflourecence Assay (IFA) method as a gold standard of ANA test, because it can help the clinician to diagnose the patient more specifically and reduce risk of false positive or errorneous based on the results of the test. From the test, the clinician might see the titers and patterns ANA of the patient. $7,15,16,19$ However, only few patients are checked for
ANA with IFA method, while the rest patients only reported whether ANA is reactive or nonreactive. ${ }^{10,11}$

According to the ICAP, ANA have associated with some autoimmune diseases which show based on the staining pattern. Homogeneous patterns was common in SLE, drug-induced lupus, and Juvenile Idiopathic Arthritis (JIA) patients, while speckled was common in Mixed Connective Tissue Disease (MCTD), SLE, Sjogren Syndrome (SJS), and Systemic Sclerosis (SSc) patients. ${ }^{15,17}$ In this study, ANA pattern of the patients only homogeneous, speckled, and nucleolar. The most frequent pattern was homogeneous (56.8\%) followed by speckled $(36.4 \%)$, and nucleolar $(6.8 \%)$. This is similar with the study of Yilmaz $\mathrm{O}$ et al. which reported homogenous pattern were the most common patterns, the result of the study showed that homogenous pattern in more than half of their patients. ${ }^{13}$ But differ with the study of Ghrahani R, et al. which are speckled $(40.3 \%)$ was the most frequent of ANA pattern, followed by nucleolar (16.1\%), nucleardot $(9.7 \%)$, and combination of nucleardot with speckled (3.2\%). ${ }^{10}$ Also, Mengeloglu Z, et al. reported speckled patterns were the most common patterns. ${ }^{12}$

Frodlund $\mathrm{M}$ et al. observed that ANA is known to have a correlation with the clinical manifestations of SLE, especially in patient with positive anti-dsDNA and/or anti-Sm. The presence of anti-dsDNA was found to be associated with renal disorder, haematological involvement, and serositis, meanwhile patient with anti-Sm had the highest frequency of malar rash, oral ulcers, arthritis, and serositis. Some ANA pattern may have an association with the clinical manifestation of SLE. Organ damage was less common among patients with speckled pattern. However, this finding needs more confirmation by others. ${ }^{18}$

There is no association between ANA pattern with the proteinuria events in this study. This result is similar with the study of Ghrahani R, et al. which stated that there was no association between ANA pattern

Table 3 Association between ANA Pattern and Proteinuria Events

\begin{tabular}{|c|c|c|c|c|}
\hline \multirow{2}{*}{ Category } & \multicolumn{2}{|c|}{ Proteinuria } & \multirow{2}{*}{$\mathbf{p}$} & \multirow{2}{*}{$\begin{array}{c}\text { OR } \\
(95 \% \mathrm{CI})\end{array}$} \\
\hline & Positive & Negative & & \\
\hline \multicolumn{5}{|l|}{ ANA Pattern $(n=44)$} \\
\hline Homogenous & 15 & 12 & $0.831^{*}$ & $\begin{array}{c}0.875 \\
(0.256-2.989)\end{array}$ \\
\hline Non - homogenous & 10 & 7 & & \\
\hline
\end{tabular}

*chi-square test, OR: Odds Ratio, CI: Confidence Interval 
with the proteinuria events with chi-square test $\mathrm{p}=0.680 .{ }^{10}$ It can be said that there is no difference with the studies even though being conducted in the different periods and the same place. Damoiseaux J, et al stated that there are several reasons for not finding an association between the ANA pattern and the manifestations of the disease. First, determination of the patterns in laboratories is inconsistent because of the subjectivity and experienced of the staff in interpretation the results. Second, healthy individual may have autoantibodies. Third, the result of the patterns may slightly differ depending on the substrate used..$^{14,17}$

This study had several limitations, the

\section{References}

1. Vinay K, Abul KA, Jon CA. Robbins and cotran pathologic basis of disease. 9th edition. Philadelphia: Elsevier Saunders. 2014. p. 211226.

2. Lehman TJA, Taber SF, Nuruzzaman F. Systemic lupus erythematosus in childhood and adolescence. in: dubois' lupus erythematosus and related syndromes. 9th ed. Elsevier Inc. 2018. p. 520-531.

3. Samanta M, Nandi M, Mondal R, Hazra A, Sarkar S, Sabui T, et al. Childhood lupus nephritis: 12 years of experience from a developing country's perspective. Eur J Rheumatol. 2017;4(3):178183.

4. Kasjmir YI, Handono K, Wijaya LK, Hamijoyo $\mathrm{L}$, Albar Z, Kalim $\mathrm{H}$, et al. Rekomendasi Perhimpunan Reumatologi Indonesia untuk diagnosis dan pengelolaan lupus eritematosus sistemik. 2011. p. 1-54.

5. Almaani S, Meara A, Rovin BH. Update on lupus nephritis - nephrology rounds. Clin J Am Soc Nephrol. 2017;12:825-35.

6. Szymanik-Grzelak H, Kuźma-Mroczkowska E, Małdyk J, Pańczyk-Tomaszewska M. Lupus nephritis in children-10 years' experience. Cent Eur J Immunol. 2016;41(3):248-54.

7. Lam N-CV, Ghetu M V, Bieniek ML. Systemic lupus erythematosus: Primary care approach to diagnosis and management. Am Fam Physician. 2016;94(4):284-94.

8. Sinha R, Raut S. Pediatric lupus nephritis: Management update. World J Nephrol. 2017;3(2):16.

9. Zhu J, Wu F, Huang X. Age-related differences in the clinical characteristics of systemic lupus data was collected retrospectively and not all samples have the variables needed (i.e. Anti Nuclear Antibody Pattern),

Based on this study, we concluded that ANA can be checked by IFA. By the test, clinician might see the different types of ANA through staining pattern. In this study, homogenous pattern was the most frequent ANA pattern in patient with SLE. Renal involvement was common in children and adolescents compared to adults patient with SLE, besides hematological involvement. Involvement of the renal can cause inflammation and one of the result is the presence of proteinuria. The homogeneous pattern has no association with proteinuria events. erythematosus in children. Rheumatol Int. 2013;33(1):111-15.

10. Ghrahani R, Setiabudiawan B, Sapartini G. Karakteristik Pola Antibodi Antinuklear pada Anak dengan Systemic Lupus Erythematosus serta Komplikasinya. J Indon Med Assoc. 2012;62(7:)273-76.

11. Gomez RLA, Uribe U, Uribe O, Romero HG, Cardiel MH, Wojdyla D, et al. Childhood systemic lupus erythematosus in Latin America: The GLADEL Experience in 230 children. Lupus. 2008;17(6):592-604.

12. Mengeloglu Z, Tas T, Kocoglu E, Aktas G, Karabork S. Determination of anti-nuclear antibody pattern distribution and clinical relationship. Pak J Med Sci. 2014;30(2):380-3.

13. Yilmaz O, Karaman M, Ergon MC, Bahar IH, Yulug N. Comparison of indirect immunoflourescence and enxyme immunoassay methods for the determination of antinuclear antibodies. Mikrobiyol Bul. 2014;38(1-2):85-90.

14. Damoiseaux J, Andrade LEC, Carballo OG, Conrad K, Francescantonio PLC, Fritzler MJ, et al. Clinical relevance of HEp-2 Indirect immunoflourescent patterns: the International Concensus on ANA Patterns (ICAP) perspective. Ann Rheum Dis. 2019;78:879-89.

15. Damoiseaux J, Andrade LEC, Carballo OG, Conrad K, Francescantonio PLC, Fritzler MJ, et al. International consensus on ANA patterns (ICAP): the bumpy road towards a consensus on reporting ANA results. 2016;7(1);1-8.

16. Cruvinel WdM, Andrade LEC, von Mühlen CA, Dellavance A, Ximenes AC, Bichara CD, et al. V Brazilian consensus guidelines for detection 
of anti-cell autoantibodies on hep-2 cells. Adv Rheumatol. 2019;59(1):28.

17. Chan EKL, Damoiseaux J, Cruvinel WdM, Carballo OG, Conrad K, Francescantonio PLC, et al. Report on the second International Consensus on ANA Patterns (ICAP) workshop in Dresden 2015. Lupus. 2016;25:797-804.

18. Frodlund M, Dahlstrom O, Kastbom A, Skogh T, Sjowall C. Associations between antinuclear antibody staining patterns and clinical features of systemic lupus erythematosus: analysis of a regional Swedish register. BMJ Open. 2013;3(6):1-8.

19. Ricchiuti V, Adams J, Hardy DJ, Katayev A, Fleming JK. Automated Processing and Evaluation of Anti-Nuclear Antibody Indirect Immunofluorescence Testing. Front Immunol. 2018;9:927. 